EXTENDED REPORT

\title{
Adenoviral p53 gene transfer inhibits human Tenon's capsule fibroblast proliferation
}

\author{
K T M Johnson, F Rödicker, K Heise, C Heinz, K-P Steuhl, B M Pützer, T Hudde
}

Br J Ophthalmol 2005;89:508-512. doi: 10.1136/bjo.2004.051664

See end of article for authors' affiliations

Correspondence to

Correspondence to:
Tobias Hudde, Zentrum für Augenheilkunde,

Abteilung für

Erkrankungen des vorderen Augenabschnitts,

Hufelandstrasse 55

D-45147 Essen, Germany; tobias.hudde@

uni-essen.de

Accepted for publication 1 September 2004
Background/aim: Although antiproliferative drugs have been used successfully to prevent scarring after filtration surgery in patients with glaucoma, complications associated with their use (such as hypotony or endophthalmitis) energise the search for an alternative treatment. Single application of $\beta$ radiation leads to long term growth arrest and expression of p53 in human Tenon's capsule fibroblasts (hTf). The authors assume that the activation of p53 is one of the cellular triggers. Their aim was to analyse the effect of p53 overexpression on $\mathrm{hTf}$ and to determine which pathways are involved.

Methods: A recombinant adenoviral vector (rAd.p53) containing transgenes encoding for human p53 and green fluorescent protein (GFP) was used to induce overexpression of p53 in hTF and a control vector (rAd.GFP). Transgene expression was detected by western blot (p53 and p21 WAF-1/Cipl $)$. Cell proliferation and viability were investigated using cell counts, 5'-bromodeoxyuridine incorporation (BrdU assay) and tetrazolium reduction (MTT assay).

Results: Infection of hTf with rAd.p53 resulted in significant inhibition of cell proliferation, DNA synthesis, and metabolic activity in vitro. Western blot showed increased levels of p53 and p21 WAF-1/Cipl in rAd.p53 infected cells, but not in rAd.GFP and uninfected cells. Apoptosis was excluded with flow cytometry.

Conclusions: Adenoviral p53 gene transfer leads to significant growth inhibition in hTf. P53 induces p2 $1^{\text {WAF-1/Cipl }}$ expression and does not cause apoptosis in hTf in vitro. p53 as an antiproliferative drug has the potential to replace mitomycin $C$ and 5 -fluorouracil in glaucoma surgery. a glaucoma surgery an additional drainage pathway for the aqueous humour is normally created to reduce intraocular pressure. The postoperative healing response can render the desired outcome of such surgery ineffective when scar formation increases outflow resistance of the artificially created drainage pathway. Antimetabolites such as 5fluorouracil or mitomycin $\mathrm{C}$ have been shown to be clinically effective at preventing bleb failure after filtration surgery, and recently in non-penetrating glaucoma surgery. ${ }^{1}$ The antifibrotic effect of those antimetabolites has been shown to derive mostly from the inhibition of human Tenon's capsule fibroblast (hTf) proliferation, ${ }^{2}$ as well as from apoptotic cell death. ${ }^{3}$ Complications associated with the use of antimetabolites, such as bleb leaks that might lead to endophthalmitis, and chronic hypotony promote the search for alternative treatments. ${ }^{5}$ We have demonstrated that mycophenolate mofetil inhibits hTf proliferation by guanosine depletion ${ }^{78}$ and could be useful clinically. Gene therapy might provide an alternative long term antiscarring adjuvans for glaucoma surgery without the complications associated with antimetabolites. ${ }^{9}$

Constable et $a l^{10}$ found that a single applications of $\beta$ radiation increased expression of p53 in hTf and they assumed that the activation of p53 is one of the cellular triggers that leads to growth arrest following irradiation. They found that growth inhibiting doses of $\beta$ radiation did not inhibit fibroblast migration or contraction. ${ }^{11}$ Since all types of ocular tissue are readily accessible for gene transfer it seems promising to bypass handling radioactive substances and to induce overexpression of p53 directly via gene transfer in order to inhibit proliferation of hTf and keep scarring at a minimum.

Since it has become clear that more than $50 \%$ of human malignant tumour cells possess mutations in p53, the gene p53 itself and its product have become the focus of intensive study. ${ }^{12}$ The tumour suppressor gene p53 is a single copy gene localised on the short arm of chromosome 17. It encodes for a phosphoprotein that, because of its short half life of about 20 minutes, is present at very low levels in the nucleus of normal cells. Human p53 contains 393 amino acids and can be divided structurally and functionally into four domains. It is a transcription factor, enhancing-upon activation-the rate of transcription of six or seven known genes that carry out functions in a cell that have become known as p53 dependent. In order to be activated for sequence specific binding to DNA, p53 is required to undergo structural change in the basic $\mathrm{C}$ terminal domain. ${ }^{12}$

Upon physical or chemical DNA damage, functional p53 may either arrest cell cycle progression in the late Gl phase, thus allowing the DNA to be repaired before its replication, or-if the damage is too extensive to be repaired-induce apoptosis. Growth arrest is achieved by the transactivational properties of $\mathrm{p} 53$, which activate a series of genes involved in cell cycle arrest. ${ }^{13}$ For p53 mediated G1 arrest p2 $1^{\text {WAF-1/Cip1 }}$ appears to be essential. ${ }^{14-18}$ In a rabbit ${ }^{9}$ and recently in a primate $^{19}$ model p $21^{\text {WAF-1/Cipl }}$ gene transfer has been shown to effectively prevent filtration surgery from failure. Adenovirus is a double strain DNA virus that has been proved to be a sufficient vector for gene transfer to many ocular cells. ${ }^{20}$ Among its advantages are its low pathogenicity and its ability to infect a broad variety of different types of cells-including post-mitotic cells. It is also possible to produce highly purified solutions of the vector, containing

Abbreviations: BrdU, bromodeoxyuridine; CDK, cyclin dependent kinases; DMEM, Dulbecco's modified Eagle's medium; ECL, enhanced chemiluminescence; GFP, green fluorescent protein; FCS, fetal calf serum; hTf, human Tenon's capsule fibroblasts; MOI, multiplicity of infection; PCNA, proliferating cell nuclear antigen; pfu, plaque forming units; rAd.p53, recombinant adenoviral vector 


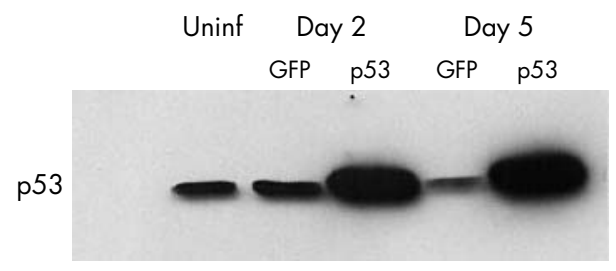

Figure 1 Western blot. Adenoviral gene transfer of p53 (rAd.p53) in human Tenon's capsule fibroblasts (hTf) leads to a marked overexpression of p53 compared to uninfected cells and cells infected with control vector (rAd.GFP).

up to $10^{12}$ plaque forming units (pfu) per ml. Genes carried by first generation adenoviral vectors into ocular tissues appear to be expressed for durations ranging from 1 day to 3 months with a maximal expression after 2-7 days. Helper dependent adenoviral vectors may lead to much longer gene expression..$^{21} 22$

In this paper we have investigated the effects of adenovirus mediated overexpression of p53 on hTf proliferation in vitro and determined its action in downstream pathways.

\section{MATERIALS AND METHODS}

\section{Cell culture of hTf}

Cell culture of hTf has been described in detail. ${ }^{7}$ Human Tenon's capsule fibroblasts (hTf) were propagated from tissue, obtained from patients undergoing routine cataract surgery. The tenets of the Declaration of Helsinki were followed and the patients' consent was obtained. Cells were grown as monolayers in Dulbecco's modified Eagle's medium (DMEM) supplemented with $10 \%$ fetal calf serum (FCS; Seromed, Berlin, Germany), penicillin (100 units $/ \mathrm{ml}$ ), streptomycin $(100 \mu \mathrm{g} / \mathrm{ml})$, and D-glucose $(4.5 \mathrm{mg} / \mathrm{ml})$. Cells were grown at $37^{\circ} \mathrm{C}$ in a humidified incubator with $10 \% \mathrm{CO}_{2}$.

\section{Construction of recombinant adenoviral vector}

We used a replication deficient adenovirus (rAd.p53), which contained genes encoding human p53 and jellyfish green fluorescent protein (GFP) as a reporter gene; and a replication deficient adenovirus, which contained only the GFP transgene (rAd.GFP). Recombinant viruses were generated using the AdEasy system ${ }^{23}$ (kindly provided by $\mathrm{B}$ Vogelstein) and have been described previously. ${ }^{24}$ Viruses were propagated, purified, and titrated as described earlier.

\section{Time course and dose finding}

In preliminary time course and dose finding experiments, hTf were infected at a multiplicity of infection (MOI) of

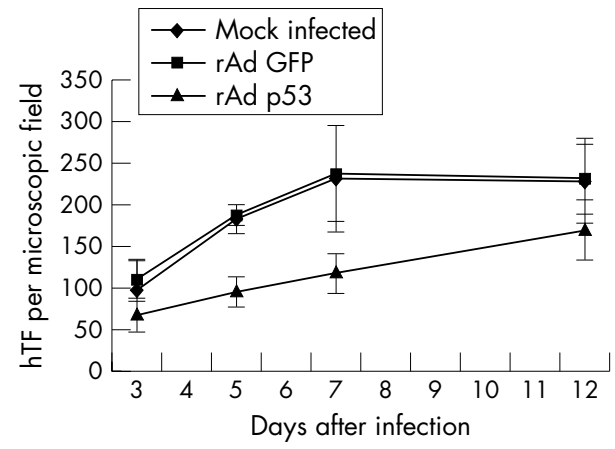

Figure 2 Cell counts per microscopic field (200x magnification) in mock infected, rAd.GFP and rAd.p53 infected hTf after alum haematoxylin staining. Gene transfer of p53 leads to significant inhibition of cell growth in hTf.
50-500 pfu per cell and were observed for up to 21 days. We calculated the percentages of GFP expressing cells by counting all fluorescent cells under an inverted fluorescence microscope and dividing that sum by the total number of cells determined using the phase contrast function of the microscope in the same field.

\section{Immunoblotting (western blot)}

For western blot analysis, we sowed out hTf at $3 \times 10^{5}$ cells per flask and infected them at MOI 100 with rAd.p53 or rAd.GFP. Uninfected cells were used as a control. Two and 5 days following infection, we prepared cell lysates and analysed protein levels essentially as described. ${ }^{25}$ We then probed those samples with mouse anti-human p53 monoclonal antibody (Sc-125, Santa Cruz, CA, USA), mouse anti-human p2 1WAF1 monoclonal antibody (OP64, Oncogene). Antibody binding sites were visualised using the appropriate horseradish peroxidase conjugated secondary antibody (anti-mouse POD, SCBT, Santa Cruz, CA, USA) according to the enhanced chemiluminescence (ECL) protocol (Amersham, Freiburg, Germany).

\section{Haematoxylin staining and cell counts}

HTf were cultured on 24 well plates at $10^{5}$ cells per well, infected with 100 MOI of either vector, or mock infected. After 3-12 days, hTf were fixed with glacial ethanol/acetic acid (95:5) and subsequently stained with alum haematoxylin for 2 minutes. The cells were air dried and analysed by microscopy at $200 \times$ magnification. Cell densities were established by determining the average of five visual fields in four different wells.

\section{BrdU assay}

We performed a colorimetric immunoassay of cell proliferation using the Cell Proliferation ELISA, BrdU (colorimetric) Kit (Roche Diagnostics, Penzberg, Germany). The assay was performed according to the manufacturer's instructions. The test is based on measuring BrdU incorporation during DNA synthesis. Quadruple samples of hTf were cultured on 96 well plates and were infected with 100 MOI of either of the two vectors, or were mock infected. After 2, 4, 6, and 10 days, wells were incubated in a medium containing BrdU for 20 hours. The reaction was stopped after 20 minutes using $25 \mu \mathrm{l} 2 \mathrm{~N} \mathrm{H}_{2} \mathrm{SO}_{4}$ per well and absorbance was measured at $450 \mathrm{~nm}$ (reference wavelength: $620 \mathrm{~nm}$ ).

\section{MTT cell viability assay}

Cell viability was examined by the MTT cell proliferation kit (ATCC, Manassas, VA, USA) following the instructions of the manufacturer. The assay is based on measuring the reduction

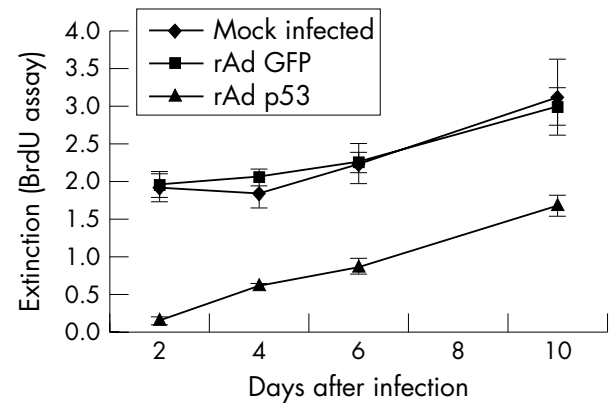

Figure $3 \mathrm{BrdU}$ incorporation in mock infected, rAd.GFP and rAd.p53 infected cells. Extinctions in the rAd.p53 samples were significantly reduced. Gene transfer of p53 leads to a marked reduction of DNA synthesis in hTf. 
of yellow tetrazolium (3-(4,5-dimethylthiazolyl-2-)-2,5diphenyltetrazolium bromide, MTT) to a purple formazan as facilitated by dehydrogenases of metabolically active cells. The intracellular formazan can be solubilised and quantified by spectrophotometric means. Quadruple samples of hTf were grown on 96 well plates and were infected with 100 MOI of either of the two vectors, or were mock infected. After $2,4,6,8$, and 14 days, wells were incubated in a medium containing yellow tetrazolium (3-(4,5-dimethylthiazolyl-2-)2,5-diphenyltetrazolium bromide, MTT) for 20 hours.

\section{Flow cytometry}

To quantitate apoptosis, we grew hTf on T25 cell culture flasks and infected at MOI 100 as described. Cells were harvested 24 hours and 72 hours after infection, fixed in 70\% ethanol and stained for DNA content with propidium iodide. Flow cytometric measurements were performed in a FACS Vantage sorter (Becton Dickinson, Heidelberg, Germany) and analysed using CelQuest software (Becton Dickinson).

\section{RESULTS}

\section{Time course and dose finding}

Preliminary time course experiments with an MOI ranging from $50 \mathrm{pfu}$ to $500 \mathrm{pfu}$ revealed the highest percentage of cells expressing GFP between 3 and 7 days after infection. Three days after infection 38\% of hTf expressed GFP at a MOI of 50 . At MOI $75,63 \%$ of the cells stained positive. At MOI 100 and 250 , nearly all cells ( $85 \%$ to $90 \%$, respectively) were found to express the reporter gene. At MOI 500, all cells were GFP positive, but cell proliferation was reduced not only in the rAd.p53 infected wells, but also-although less markedin the rAd.GFP group. This result is apparently due to the cytotoxic effect of adenoviral vector at this high virus dose (data not shown). We therefore decided to use MOI 100 for all following experiments.

\section{Immunoblot for p53}

With immunoblotting (western blot) for p53, we then searched for the gene product of the transgene p53. Even though low levels of p53 could be detected in all samples of hTf, vector induced overexpression was observed in all rAd.p53 infected samples between days 2 and 5 following infection, but not in the rAd.GFP and the uninfected sample (fig 1).

Computer assisted quantification of $\mathrm{p} 53$ revealed that bands of rAd.p53 infected cells at days 2 and 5 after infection were between 3 and 3.7 times stronger than in the uninfected control. At the same time the bands of rAd.GFP infected cells varied only slightly.

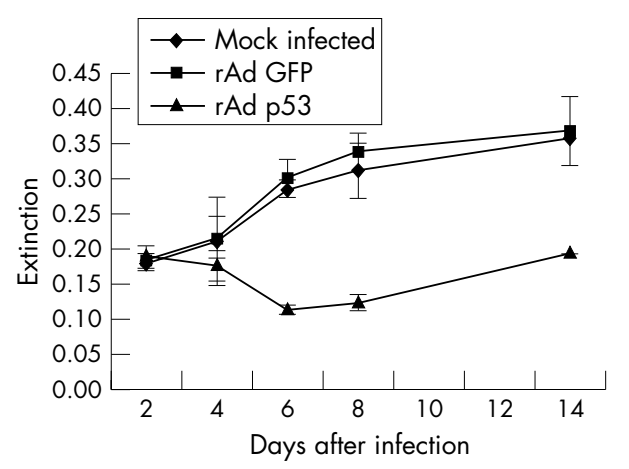

Figure 4 MTT cell viability assay of mock infected, rAd.GFP and rAd.p53 infected hTf. Extinctions were significantly reduced in rAd.p53 infected cells, but not in rAd.GFP and mock infected cells. Gene transfer of p53 leads to a marked decrease of metabolic cell activity.

\section{Cell counts}

To examine cell proliferation by cell numbers we counted haematoxylin stained hTf in 24 wells per microscopic visual field (magnification $200 \times$ ). Untreated and rAd.GFP infected cells grew continuously (from 97.6 to 182.75, mock infected; and from 109.2 to 188 , rAd.GFP infected) reaching a plateau at about 230 on day 8. Numbers of rAd.p53 treated cells significantly lagged behind both rAd.GFP and mock infected controls. Here the cell numbers grew more slowly from 67 to 95.25 on day 5 and to 117.5 on day 7. HTf continued to grow until they reached an average cell count of 169.75 on day 12 (fig 2).

\section{BrdU assay}

The BrdU assay measures incorporation of BrdU in cell DNA which correlates with DNA synthesis and allows a direct evaluation of cell proliferation on DNA level. Absorbances of rAd.GFP infected and mock infected cells did not differ significantly, indicating that the adenoviral vector itself has no significant effect on cell proliferation. Both curves show a steady increase over the 10 days following treatment. Absorbance in rAd.p53 infected cells was significantly below both controls, even though the difference between those cells and both controls appeared to diminish (fig 3).

\section{MTT cell viability assay}

The MTT cell viability assay is commonly used to analyse metabolic activity in proliferating cells. It measures the reduction of tetrazolium salts facilitated by mitochondrial dehydrogenases. Thus, a multitude of factors such as cell number, cell metabolism, mitochondrial activation, etc, influence the outcome of this assay. Absorbances of mock infected and rAd.GFP infected hTf did not differ essentially indicating that the adenoviral construct itself does not influence cell metabolism significantly at MOI 100. Both curves showed exponential to linear increase during the 14 days following treatment. Absorbance in rAd.p53 infected cells did diminish until the sixth day after infection. Nevertheless, it seemed to gain on both controls during days 8-14 (fig 4).

\section{Flow cytometry}

Flow cytometry classified $80 \%$ to $90 \%$ of all gated cells in all samples as Gl. The sub-Gl percentage of untreated hTf was measured as $9.37 \%$ of all gated cells and neither rAd.GFP (with $3.51 \%$ and $3.12 \%$ sub-Gl cells on days 1 and 3 following infection), nor rAd.p53 (with $1.47 \%$ and $5.76 \%$ subGl cells on days 1 and 3 following infection infected cells) exceeded this finding (fig 5). As a consequence, we can conclude that p53 expression in these cells is not associated with an increase in the sub-Gl population, indicative for apoptosis.

\section{Immunoblot for p2 1 WAF-1/Cip 1}

Levels of p2 $1^{\text {WAF-1/Cip1 }}$ in rAd.p53 infected cells were shown to be elevated on days 2 to 5 following infection (fig 6). Computer assisted quantification of p2 IWAF1 bands revealed a clear increase of p21 in rAd.p53 infected cells at days 2 and 5 compared to rAd.GFP treated cells.

\section{DISCUSSION}

Approaches to modify the wound healing response after filtering surgery by oligonucleotide or gene transfer have been reported by different research groups. Akimoto et $a^{26}$ blocked the transcription factor E2F in hTf by transferring double stranded phosphorothioate oligonucleotides containing E2F transcription factor cis element with the haemagglutinating virus of Japan cationic liposomes and found growth inhibition. In later work, Akimoto et al ${ }^{27}$ constructed an 


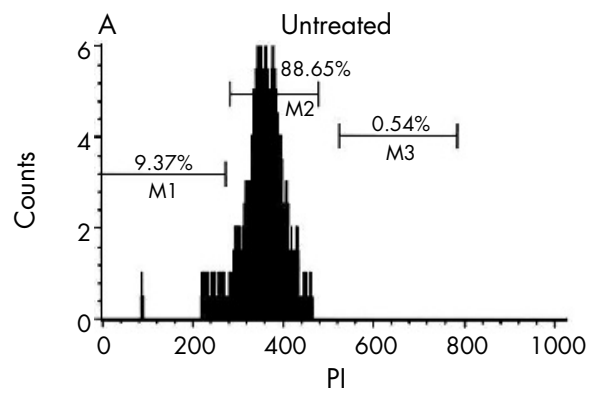

Figure 5 Flow cytometry to quantitate apoptosis was performed in rAd.p53 infected, rAd.GFP infected and untreated hTf after propidium iodide staining. No significant difference in the sub-Gl percentage was detected indicating no significant induction of apoptosis by $\mathrm{p} 53$ gene transfer.
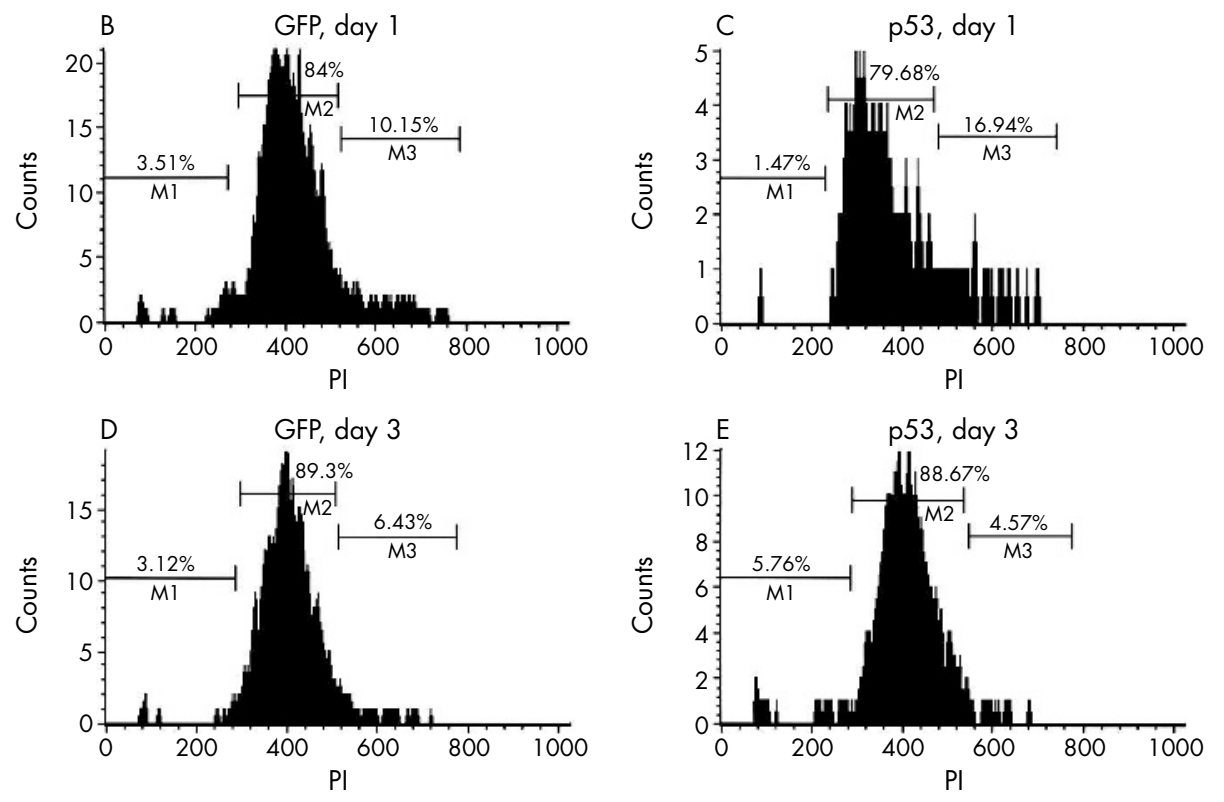

adenoviral vector to transfer a gene encoding for cytosine deaminase in rabbit Tenon fibroblasts. If the pro-drug 5 -fluorocytosine was co-exposed, it was converted into 5 -fluorouracil in infected cells and inhibited cell growth. In a rabbit ${ }^{9}$ and recently in a primate ${ }^{19}$ model, p $21^{\text {WAF-1/Cipl }}$ gene transfer prevented failure of filtration surgery. For p53 mediated G1 arrest, p2 $1^{\text {WAF-1/Cipl }}$ appears to be essential. ${ }^{14-18}$ In normal cells this $21 \mathrm{kDa}$ product of the WAFl gene is found in a complex of cyclins, cyclin dependent kinases (CDK), and the proliferating cell nuclear antigen (PCNA), where it appears to inhibit CDK activity. ${ }^{14-17}$ Among the effects of $\mathrm{p} 2 \mathrm{1}^{\mathrm{WAF}-\mathrm{1} / \mathrm{Cipl}}$ mediated CDK inhibition is the prevention of the CDK dependent inactivation of the product of the retinoblastoma gene. This makes p21 2 WAF-1/Cipl a powerful, but reversible inhibitor of cell cycle progression at both G1 and G2, ${ }^{14-16}{ }^{18}$ which apparently allows DNA repair to happen. Constable et $a l^{10}$ showed that a single application of $\beta$ radiation increased the expression of p53 in hTf and they assumed that the activation of $\mathrm{p} 53$ is one of the cellular

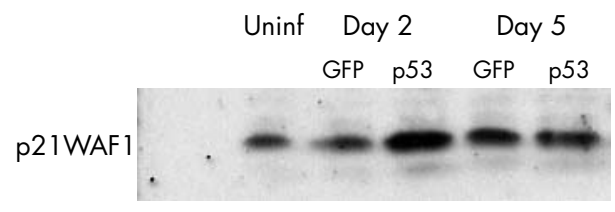

Figure 6 Western blot shows that $\mathrm{p} 53$ gene transfer leads to an increased expression of $\mathrm{p} 21^{\mathrm{WAFl} / \mathrm{CIPl}}$ in hTf compared to uninfected and rAd.GFP infected cells. The observed effect of p53 gene transfer on hTf cell growth appears to be mediated by $\mathrm{p} 21^{\mathrm{WAFl} / \mathrm{Cipl}}$ and not by apoptosis. triggers that leads to growth arrest following irradiation. Interestingly, growth inhibiting doses of $\beta$ radiation did not inhibit fibroblast migration or contraction. ${ }^{11}$ In this work, we demonstrate that adenoviral transfer of p53 induces a marked overexpression of p53 in hTf. This leads to a significant inhibition of cell proliferation, DNA synthesis, and metabolic cell activity. The absence of any such effect in controls leads us to assume that this effect is mediated by p53 and its downstream proteins. Using flow cytometry we could not detect any significant increase in apoptosis in our in vitro model. Furthermore, we could demonstrate that overexpression of $\mathrm{p} 53$ leads to increased expression of $\mathrm{p} 21^{\mathrm{WAF}-\mathrm{1} / \mathrm{Cipl}}$ in hTf. It can therefore be assumed that p53 enhanced transcription at the p2lWAF gene, which in turn leads to p2 IWAFl dependent inhibition of CDK and PCNA mediated DNA replication, whereas apoptotic pathways did not appear to be of any consequence.

At MOI 500, the recombinant adenoviral vector itself interferes with cell growth (data not shown), which adds to the effect of p53. We therefore used MOI 100, although only $85 \%$ of hTf expressed the reporter gene GFP. Still, the effects were significant.

p53 shows promise as an antiproliferative drug to regulate wound healing following glaucoma surgery. It inhibits cell proliferation and might thus prevent excessive scarring without inducing apoptosis, as is the case with antimetabolites such as mitomycin $\mathrm{C}$ or $5^{\prime}$-fluorouracil. ${ }^{28} \mathrm{~T}$ cell augmented cell death of mitomycin $\mathrm{C}$ treated hTf is discussed as a cause of hypocellular drainage blebs in patients who have undergone glaucoma surgery. ${ }^{3}$ Apoptosis free inhibition of cell growth by adenoviral transfer of p53 could avoid this particular troublesome side effect. p53 gene transfer probably 
does not interfere with other clinically important cell functions such as migration, contraction, and production of extracellular matrix. Investigating the effect of p53 on those functions might prove to be a promising field for further studies. ${ }^{10} 11$

\section{ACKNOWLEDGEMENTS}

This work was supported by Deutsche Forschungsgemeinschaft grant $\mathrm{Hu} 761 / 2-1$ and by an IFORES Promotionsstipendium (KTM Johnson).

\section{Authors' affiliations}

K T M Johnson, K Heise, C Heinz, K-P Steuhl, T Hudde, Zentrum für Augenheilkunde, Abteilung für Erkrankungen des vorderen Augenabschnitts, Hufelandstrasse 55, D-45147 Essen, Germany F Rödicker, B M Pützer, Department of Vectorology and Experimental Gene Therapy, University of Rostock Medical School, Institute Bldg, Schillingallee 70, D-18055 Rostock, Germany

None of the authors has any commercial interest in any of the products or methods mentioned or any other competing interest.

\section{REFERENCES}

1 Kozobolis VP, Christodoulakis EV, Tzanakis N, et al. Primary deep sclerectomy versus primary deep sclerectomy with the use of mitomycin $C$ in primary open-angle glaucoma. J Glaucoma 2002;11:287-93.

2 Khaw PT, Ward S, Porter A, et al. The long-term effects of 5-fluorouracil and sodium butyrate on human Tenon's fibroblasts. Invest Ophthalmol Vis Sci 1992;33:2043-52.

3 Crowston JG, Chang LH, Daniels JT, et al. T lymphocyte mediated lysis of mitomycin C treated Tenon's capsule fibroblasts. Br J Ophthalmol 2004;88:399-405.

4 Crowston JG, Akbar AN, Constable PH, et al. Antimetabolite-induced apoptosis in Tenon's capsule fibroblasts. Invest Ophthalmol Vis Sci 1998;39:449-54.

5 Wolner B, Liebmann JM, Sassani JW, et al. Late bleb-related endophthalmitis after trabeculectomy with adjunctive 5-fluorouracil. Ophthalmology 1991;98:1053-60.

6 Mandal AK, Prasad K, Naduvilath TJ. Surgical results and complications of mitomycin C-augmented trabeculectomy in refractory developmental glaucoma. Ophthalmic Surg Lasers 1999;30:473-80.

7 Heinz C, Heise K, Hudde T, et al. Mycophenolate mofetil inhibits human Tenon fibroblast proliferation by guanosine depletion. $\mathrm{Br} J$ Ophthalmol 2003;87:1397-8.

8 Heinz C, Hudde T, Heise K, et al. Antiproliferative effect of mycophenolate mofetil on cultured human Tenon fibroblasts. Graefes Arch Clin Exp Ophthalmol 2002;240:408-14.
9 Perkins TW, Faha B, Ni M, et al. Adenovirus-mediated gene therapy using human p21WAF-1/Cip-1 to prevent wound healing in a rabbit model of glaucoma filtration surgery. Arch Ophthalmol 2002;120:941-9.

10 Constable PH, Crowston JG, Occleston NL, et al. Long term growth arrest of human Tenon's fibroblasts following single applications of beta radiation. Br J Ophthalmol 1998;82:448-52.

11 Constable PH, Crowston JG, Occleston NL, et al. The effects of single doses of $\beta$ radiation on the wound healing behaviour of human Tenon's capsule fibroblasts. Br J Ophthalmol 2004;88:169-73.

12 Levine AJ. p53, the cellular gatekeeper for growth and division. Cell 1997;88:323-31

13 Soussi T, Dehouche K, Beroud C. p53 website and analysis of p53 gene mutations in human cancer: forging a link between epidemiology and carcinogenesis. Hum Mutat 2000;15:105-13.

14 El-Deiry WS, Harper JW, O'Connor PM, et al. WAF1/CIP1 is induced in p53mediated G1 arrest and apoptosis. Cancer Res 1994;54:1169-74.

15 El-Deiry WS, Tokino T, Velculescu VE, et al. WAF1, a potential mediator of p53 tumor suppression. Cell 1993;75:817-25.

16 El-Deiry WS, Tokino T, Waldman T, et al. Topological control of p21WAF1 / CIP1 expression in normal and neoplastic tissues. Cancer Res 1995;55:2910-9.

17 Xiong Y, Zhang H, Beach D. Subunit rearrangement of the cyclin-dependent kinases is associated with cellular transformation. Genes Dev 1993; 7:1572-83.

18 Agarwal R, Talati M, Lambert W, et al. Fas-activated apoptosis and apoptosis mediators in human trabecular meshwork cells. Exp Eye Res 1999:68:583-90

19 Heatley G, Kiland J, Faha B, et al. Gene therapy using p21 (WAF-1/Cip-1) to modulate wound healing after glaucoma trabeculectomy surgery in a primate model of ocular hypertension. Gene Ther 2004;11:949-55.

20 Larkin DFP, Oral HB, Ring C, et al. Adenovirus-mediated gene delivery to the corneal endothelium. Transplantation 1996;61:363-370.

21 Maione D, Wiznerowicz M, Delmastro P, et al. Prolonged expression and effective readministration of erythropoietin delivered with a fully deleted adenoviral vector. [In Process Citation]. Hum Gene Ther 2000;1 1:859-68.

22 Morral N, O'Neal W, Rice K, et al. Administration of helper-dependent adenoviral vectors and sequential delivery of different vector serotype for long-term liver-directed gene transfer in baboons. Proc Natl Acad Sci USA 1999;96:12816-21.

23 He TC, Zhou S, da Costa LT, et al. A simplified system for generating recombinant adenoviruses. Proc Natl Acad Sci USA 1998;95:2509-14.

24 Rodicker F, Putzer BM. p73 is effective in p53-null pancreatic cancer cells resistant to wild-type TP53 gene replacement. Cancer Res 2003;63:2737-41.

25 Stiewe T, Putzer BM. Role of the p53-homologue p73 in E2F1-induced apoptosis. Nat Genet 2000;26:464-9.

26 Akimoto $M$, Hangai $M$, Okazaki K, et al. Growth inhibition of cultured human Tenon's fibroblastic cells by targeting the E2F transcription factor. Exp Eye Res 1998;67:395-401.

27 Akimoto M Miyahara T, Arai J, et al. A new delivery system for 5-fluorouracil using prodrug and converting enzyme. Br J Ophthalmol 2002;86:581-6.

28 Crowston JG, Chang LH, Constable PH, et al. Apoptosis gene expression and death receptor signaling in mitomycin-C-treated human tenon capsule fibroblasts. Invest Ophthalmol Vis Sci 2002;43:692-9. 